\title{
Towards Safer Rides: Measuring Motorcycle Dynamics with Smartphones
}

\author{
M. Stanglmayr ${ }^{1 *}$, M. Bäumler ${ }^{1}$, K. Büttner ${ }^{1}$, G. Prokop ${ }^{1}$
}

\begin{abstract}
Motorcyclists are among the most vulnerable road users in road traffic. Often, the cause of accidents is a loss of control on rural roads which could be averted by making use of the physical potential in terms of larger lean angles. At the same time, in reality driven lean angles over a larger group of riders and a longer route are unknown which is mainly due to the special measuring technology required. The focus is therefore on the development of a low-cost measurement method for measuring the lean angles of motorcycles. Smartphones are usually characterized by integrated inertial sensors, which are suitable for the acquisition of motorcycle driving dynamics. Employing a smartphone app tailored to the requirements for collecting measurement data on the motorcycle, the data of the sensors are recorded. During the offline evaluation, the rotation angles between the smartphone and the motorcycle coordinate system are determined, the inertial measurement data are transformed and the roll angle is calculated. An essential part is the alignment of the developed measurement chain with a high-precision measurement system. This was carried out on different routes and thus the data quality was determined. As a feasibility study, a test person study with several participants was carried out, which confirmed the practical suitability of the measurement chain. Hence, the study outcomes are briefly shown and discussed. The successful validation on different routes, the practical suitability of the data acquisition and the accuracy of the measurement system encourage to roll out the smartphone app to a larger panel of test persons and thus to collect data on a larger driver collective.

\section{Keywords}

Motorcycle Dynamics - Smartphone - Motorcyle Safety - Data Aquisition - Coordinate Transformation - Kalman Filter - Roll Angle Estimation

${ }^{1}$ Chair of Automobile Engineering, Technische Universität Dresden, Dresden, Germany

*Corresponding author: maximilian.stanglmayr@tu-dresden.de
\end{abstract}

\section{Introduction}

As unprotected road users, motorcyclists are among the most vulnerable groups of road users [1]. Due to their naturally unstable driving dynamics, a high power-to-mass ratio and the roads to be travelled, their accident severity is above average. Additionally, motorcyclists are particularly likely to suffer serious injuries in accidents due to their small protection zone. Compared to car drivers, motorcyclists are $[2,3]$ :

- 3,9 times more often involved in a traffic accident,

- 6,7 times more often injured in a traffic accident,

- 20,6 times more often killed in a traffic accident.

Hereby, over $45 \%$ of all killed motorcyclists crash in curves, mostly caused by subjectively too high perceived speed [4]. However, the speed and thus the number of accidents could often be reduced by increasing the corresponding lean angle.
In general, the reasons for driven lean angles seem to be diverse. Bauer et al. motivate that motorcyclists do not take advantage of the physical limit of their vehicles [4]. Spiegel [5] anticipates that a natural lean angle limit of $20^{\circ}$ exists. Others correlate driven lean angles with rider characteristics: Winkelbauer et al. with annual mileage [6], Hädrich with driving experience in years [7] and Praschl et al. with rider type (e.g. from sporty to comfort-oriented) [8]. Nevertheless, there exists no systematic review on the reasons for specific lean angle behavior of motorcyclists, despite research methods exist.

In addition to motorcycle riding simulators (e.g. [9]), naturalistic riding studies (NRS) are one way of investigating driven lean angles (e.g. [10]). Up to now, the latter is mainly based on motorcycles equipped with special measurement technology. While measurement-motorcycle-studies en-

\footnotetext{
This work is licensed under the Creative Commons Attribution-NonCommercial 4.0 International (CC BY-NC 4.0). To view a copy of this license, visit https://creativecommons.org/licenses/by-nc/4.0/.
} 
sure high data quality, the rider collective to be addressed is normally quite small due to high efforts in terms of cost and administration. In contrast, smartphones are a low-cost alternative, which is both inexpensive and widely available. With over 57.7 million (69\% of the population) smartphone users in Germany and a user share of $95 \%$ in the motorcycle relevant target group up to 49 years [11], they seem to be an interesting research tool. This is also proven by a steadily increasing amount of research studies demonstrating the suitability of smartphone sensors for research purposes [12-23].

The closest research to ours was done by Kamimura et al. [24] who developed first single elements towards a complete measurement chain: A smartphone application and data filtering / data analysis components.

With focus to the measurement of driven lean angles, there are also smartphone applications such as calimoto ${ }^{\circledR 1}$ or EatSleepRide ${ }^{\circledR 2}$. However, they do not provide insight into the exact lean angle and calibration methods and thus may not be used for our research purposes.

Therefore, this paper focuses on developing a performant and low-cost smartphonemeasurement-toolchain for the investigation of driven lean angles of large rider collectives.

\section{State of Research}

Regarding the state of research, we briefly go into the basics of motorcycle lateral dynamics (1.1) and applied coordinate systems (1.2), give an overview over smartphone sensors (1.3) and how the motorcycle roll angle can be estimated from sensor data (1.4).

\subsection{Motorcycle Lateral Dynamics}

In contrast to a two-track vehicle, a motorcycle requires a lean angle to be able to negotiate a curve with the curvature $\kappa=1 / r$. While a car rolls to the outside of the curve when cornering, a motorcycle requires a roll angle towards the centre of the curve to support the centrifugal force. In the case of stationary cornering, the resultant of centrifugal force $F_{C}=m v^{2} \kappa$ and weight force $F_{G}=m g$ points through the tyre contact line where $m$ being the mass of the vehicle, $v$ the forward speed and $g$ the earth gravity constant.

An equation for the roll angle $\varphi_{t h}$ can be derived from the vectorial forces, resulting in the following relationship.

$$
F_{G} \cdot \sin \varphi_{t h}=F_{C} \cdot \cos \varphi_{t h} \Leftrightarrow \varphi_{t h}=\arctan \frac{v^{2} \cdot \kappa}{g}
$$

Equation 1 shows that at higher lateral acceleration $v^{2} \kappa$ or higher speed, a larger roll angle is

\footnotetext{
${ }^{1}$ calimoto $\mathrm{GmbH}$, Potsdam, Germany

${ }^{2}$ Kissaki Red Inc. of Toronto, Ontario, Canada
}

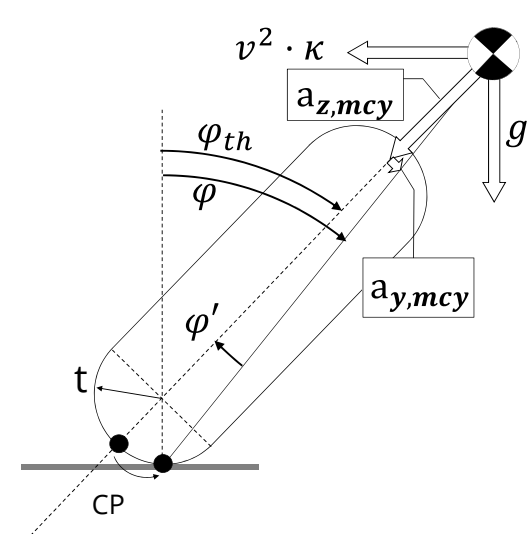

Illustration of Eq. 1 under the assumption of infinitely narrow, undeformed tyres (therefore $\varphi_{t h}$ ) and the neglect of gyroscopic forces. The effective roll angle $\varphi$ is the result when taking into account the tirewidth $t$.

Figure 1. Ideal and real roll angle; inspired by [25]

necessary to pass the curve. Due to the tire width, which is finite in reality, the tire contact point on the tire surface moves towards the center of the curve during cornering. Therefore, a larger roll angle must be set, which is composed of the physically effective rolling angle $\varphi_{t h}$ and an additional angle $\varphi^{\prime}$. Figure 1 illustrates both the theoretical and geometric total roll angle.

The geometric total roll angle $\varphi$ is therefore calculated by adding the physically effective roll angle $\varphi_{t h}$ and the additional roll angle $\varphi^{\prime}$. While Cossalter [25] gives an equation that takes into account the additional roll angle due to tire width (Eq. 3), the additional roll angle could on average be simplified to approx. $10 \%$ [26] - see Eq. 3.

$$
\begin{array}{r}
\varphi=\varphi_{t h}+\varphi^{\prime}=\varphi_{t h}+\arcsin \frac{t \cdot \sin \left(\arctan \frac{v^{2} \cdot \kappa}{g}\right)}{h-t} \\
\varphi=\varphi_{t h}+\varphi^{\prime} \approx 1,1 \varphi_{t h}
\end{array}
$$

Due to the relationship between centrifugal and weight forces already presented, a maximum possible drivable roll angle of $50^{\circ}$ can be achieved for dry road conditions and the assumption of a coefficient of friction of $\mu=1$. Additional components of the motorcycle like luggage, exhaust parts or footrests can lower this theoretically possible roll angle.

\subsection{Coordinate Systems}

The coordinate system used is based on DIN ISO 8855 [27]. The coordinate origin of the vehiclerelated system is located in the centre of gravity of the vehicle. The axes are described with $\left[\left(X_{m c y}, Y_{m c y}, Z_{m c y}\right)\right]^{T} . X_{m c y}$ is located in the longitudinal median plane of the vehicle and points horizontally to the front, while $Y_{m c y}$ points to the left and is vertical to this plane. Following the right-hand 
convention, $Z_{m c y}$ points upwards. Rotations around the axes $X_{m c y}$ and $Y_{m c y}$ are called rolling and pitching, and a rotation around the vertical axis $Z_{m c y}$ is called yawing. The roll angle is designated $\varphi$. The time derivation of the roll angle is called roll rate $\dot{\varphi}$.

\subsection{Smartphone Sensors}

Modern smartphones typically integrate an accelerometer, gyroscope and magnetometer as "hardware" sensors which are accompanied by a GPS receiver. Additionally, luxmeters, microphones, proximity sensors and barometers are also often available. Within the operating system, an application can retrieve sensor data over well documented APIs.

The accuracy of the sensors built into current smartphone models is something that most smartphone manufacturers provide little information about. A comparison of high-precision measurement technology and different smartphone sensors by Schelewsky et al. [12] comes to the conclusion that the variance of smartphone sensor technology has decreased considerably (71\%) over time, for example from iPhone ${ }^{3} 4$ to iPhone ${ }^{3}$. Nevertheless, there is a significant difference between precision measurement technology and the best smartphone that is compared.

A further comparison to the commercial available inertial measurement platforms from GeneSys Elektronik [28] shows that the variance of acceleration and gyro sensors between iPhone 35 and ADMA $\mathrm{G}$ differ by a factor of 2000 and 6000 . Comparing the data from Schelewsky et al. and Genesys, we find that the noise density, which describes the lower resolution limit of a sensor, is also many times higher for smartphone sensors than for high-precision sensors.

Ma et al. [29] evaluate sensor quality of mobile phone sensors and conclude that accelerometer and gyroscope sensors are rather stable and GPS deviation is not more than $10 \mathrm{~m}$ from the actual value.

Kos et al. [30] use a cloud-based data collection environment to gain more insight into sensor performance for cross-platform smartphone developers. Among other details, they state that the sensor quality between platforms varies considerably and even within the same model. That motivates the question if smartphone sensors are suitable for capturing the dynamics of motorcycle riding.

\subsection{Roll angle Estimation Methods}

The authors identified four roll angle estimation method clusters. These are 1) the frequency separation principle, 2) optical sensors, 3) Kalman Filter sensor fusion and 4) others. For now, we deliberately exclude algorithms to estimate the pose and position of bodies which are developed in the field

\footnotetext{
${ }^{3}$ Trademark of Apple Inc.
}

of inertial navigation. To determine the impact of the existing methods on the methods to be developed, the findings are concluded shortly at the end of this section.

Frequency Separation Taking into account the characteristics of motorcycle maneuvers, the frequency separation principle features the fusion of the integrated roll rate (high frequency) with a somehow calculated stationary roll angle (low frequency) values. The accordingly low- and highpass filtered channels are then added up to give a roll angle estimation.

Boniolo et al. [31] use different datasets of inertial sensors to estimate the low-frequency part with the use of a neural network. Also, through optimisation, the separating frequency is determined. The best combination of inertial data was a set of the lateral acceleration, yaw rate and forward velocity. Later, Boniolo and Savaresi [32] find that angular rates measurements also provide sufficient accuracy regarding the low-frequency component. In their patent, Ambruzs et al. [33] describe the calculation of the stationary roll angle mainly based on the lateral and vertical acceleration. This also takes into account the additional roll angle due to tire width.

Optical Sensors Two or more optical sensors which measure the distance to the ground are used. The sensors are e.g. mounted on the left and right foot peg. Through trigonometry calculations, the roll angle can be calculated. This method is often used to deliver ground-truth data in the development stage. Boniolo et al. [34] state that the optical sensor method is a cost-effective method to provide a valid roll angle signal despite that rough asphalt or solar light can reduce the accuracy of the measuring system. Additional uncertainty analysis and change of the sensor principle in [35] reveals that the weak points of the method could be eliminated. However, Lot et al. [36] still mention some problems with the optical sensor method when comparing the results to a roll angle estimation method.

Kalman Filtering Regarding roll angle estimation, Kalman filters are used to fuse signals from different sensors. Like in the frequency separation method, usually stationary and dynamic measurement channels are separately fed into the filter. The filter outputs an optimal estimation value. Corbetta et al. [37] compare Extended (EKF) and Unscented Kalman (UKF) filters and state that the UKF is more robust regarding to initialisation value uncertainties. Also Schlipsing et al. [38] use an EKF with an estimation of the additional roll angle due to tire width by setting $\varphi^{\prime}=1,11$. By deriving the state-space formulation out of a multibody system for the core of an EKF, Lot et al. [36] estimate the roll angle based on simulations with errors. Often, the Root Mean Square Error (RMSE) is used for a comparison of 
different time signals. Here, the error is about $4^{\circ}$ and $R M S E=1.5^{\circ}$. Validation on a real race track yields roll angle errors with peaks above $10^{\circ}$ and $R M S E=4^{\circ}$.

Others Different methods for roll angle estimation are the pure calculation by GPS data only [7] or the use of image processing algorithms to estimate the roll angle from a video camera [38].

Conclusion It can be concluded that the clusters "Others" and "Optical Sensors" can be omitted since no useful sensors exist on a smartphone to apply these methods. Usually, smartphones are equipped with cameras but those might in practice be occluded while riding a motorcycle. Also, GPS data from smartphones can be rather inaccurate. In a preliminary study, we identify a max. sampling frequency of $1 \mathrm{~Hz}$, which also excludes a calculation of the roll angle with GPS data only. With the "Frequency Separation Principle" and "Kalman Filters" leftover we conclude that the key feature of a successful method is a good estimation of the stationary roll angle. Regarding the dynamic part, the integration of the roll rate $\dot{\varphi}$ is well established.

To fuse both the dynamic and stationary parts, the frequency separation principle is often used. Usually, the separation frequency is set empirically or through optimisation which is one error contributor to the whole method. The authors, therefore, propose to use a simple linear Kalman Filter which itself outputs an optimal estimation of the roll angle.

\section{Methods}

The measurement chain is separated between (online) data measurement and (offline) postprocessing as can be seen in Figure 2. This means that the tailor - cut smartphone app "MotoLogger" is optimized for reliably recording raw data on the motorcycle which is then transferred via USB to a computer. The subsequent transformation, filtering, calculation and event- filtering tasks happen within a stationary MATLAB ${ }^{\circledR}$ environment. The MATLAB ${ }^{\circledR}$ environment is programmed objectoriented so that the datasets for different rides can be processed efficiently.

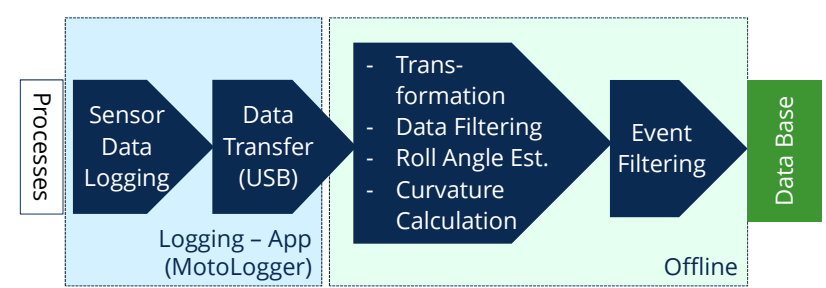

Figure 2. Topology of the measurement chain with a smartphone app and offline processing environment

\subsection{Smartphone Application}

Despite Staaks et al. [39] present a smartphone application "phyphox" for conducting educational physics experiments, the authors decided to develop a tailored Android" application, called "MotoLogger", due to several reasons. Firstly, the MotoLogger application is designed for high usability. Study-specific parameters, like age and rider-ID, can be directly retrieved from the users (see Figure 3). Moreover, the user interface was kept as simple as possible avoiding mishandling. Secondly, the measurement data are continuously written in ASCII readable files (*.CSv) at each time step for each sensor preventing data loss due to interruptions (e.g. overheating). Thirdly, the sampling rates can be set to the corresponding requirement (Gyroscope, Accelerometer $=100 \mathrm{~Hz}, \mathrm{GPS}=1 \mathrm{~Hz}$ ). Fourthly, the start of each sensor recording can be aligned. This is highly relevant since the start of GPS recording depends on the GPS-signal being available. Fifthly, the user-specific data are only saved in the internal memory to ensure data privacy. Finally, energy requirements can be set to a maximum as usually apps are required to be battery-saving and therefore restricted by the operating system. Tests with the mid-class smartphone "LG G7fit" proofed a maximum recording time of 4 hours due to battery life and a storage-consumption of $100 \mathrm{MB}$ per recording hour. By connecting the smartphone with the motorcycle's electric system and considering the built-in storage capacity of $32 \mathrm{~GB}$, the recording of a whole day-trip is possible.

\subsection{Smartphone Coordinate Transformation}

By definition, the typical orientation of an Android smartphone (short: SP) coordinate system points with the $Y$-axis towards the top edge of the screen, the $X$-axis points towards the right edge of the screen and the Z-axis points out of the screen towards the viewer. For example, a smartphone which is mounted on the motorcycle with the top edge pointing in the direction of travel and the display aiming towards the rider is therefore rotated by $a$ yaw angle of $\psi_{S P}=90^{\circ}$ and e.g. a roll angle $\varphi_{S P}=33^{\circ}$ to the vehicle coordinate system (see Figure 4).

However, different mounting methods imply that the orientation of the smartphone coordinate system to the coordinate system of the motorcycle may be different for each ride. A measurement of the respective angles to the motorcycle coordinate system would be very complex and is not practicable, especially for future test rides where the test person should also have the possibility to carry out the test independently. Consequently, a method was developed that automatically transforms the measurement data related to the smartphone coordinate system (see Figure 5) into the motorcycle 


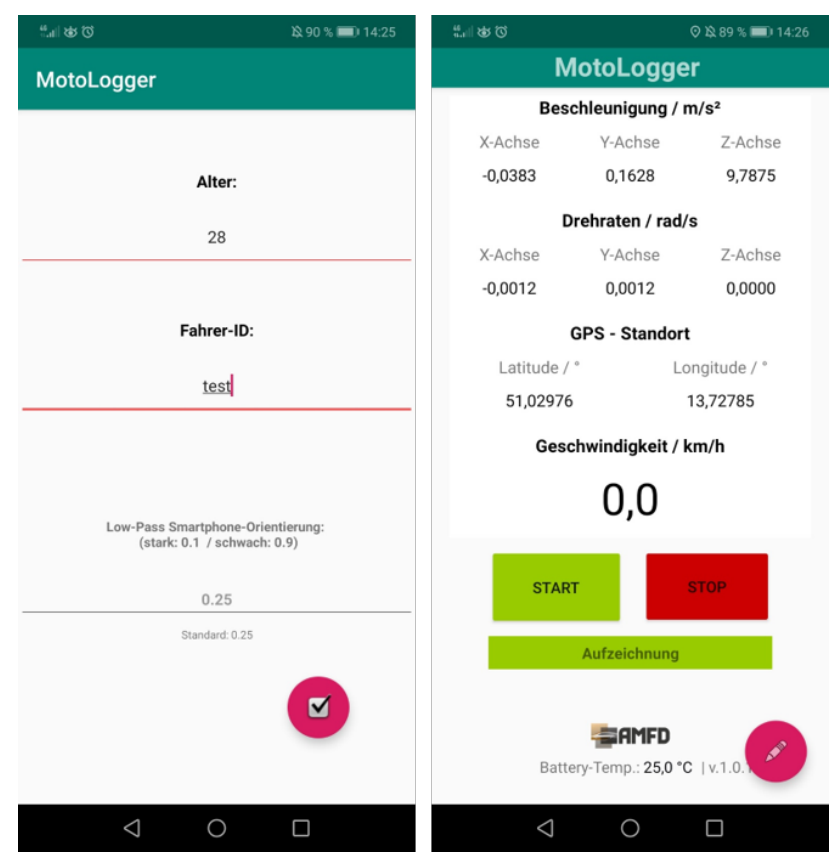

Overview of the MotoLogger app's user interface, which consists of two different windows. Left: Form for filling in rider data (age, rider code). Right: Instantaneous values, two buttons to start and stop data logging and valuable system information.

Figure 3. User interface of the "MotoLogger" application

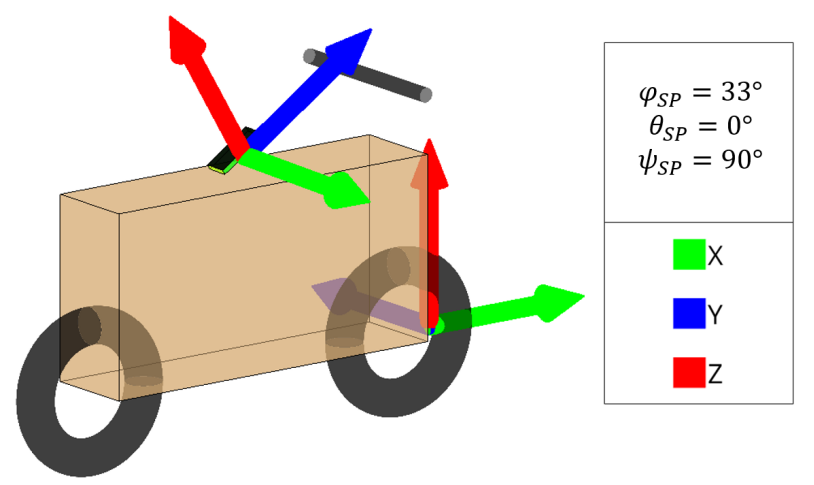

Figure 4. Rotation of coordinate systems

coordinate system. The method is separated into three major steps described below.

Step 1 Tests with various test persons have shown that an automated determination of the roll and pitch angles cannot be reliably carried out when the vehicle is standing still, as in this case it must be assumed that the vehicle is as upright as possible. As soon as the motorcycle is standing on the side stand or there is a roll angle when stationary due to a too-short leg length, the above requirement is not met and the transformation is faulty. Therefore, a datapoint selection has to be applied to find "straight maneuvers without accelerating".

The first data point selection applies if a minimum speed of $v_{\min }=30 \mathrm{~km} / \mathrm{h}$ is reached, the acceleration of $a_{\text {thresh }}=0,3 \mathrm{~m} / \mathrm{s}^{2}$ is not exceeded and the sum of the three rotation rates does not exceed a calculated threshold value. Looking at the sum of rotation rates is admissible, since at least the pitch and roll rate would be present in the motorcycle coordinate system while stationary cornering. The following Eqs. 4 - 6 are used for a calculation to determine the speed-dependent threshold value of the rotation rates:

$$
\begin{aligned}
\dot{\psi}_{g l o b} & =v \cdot \kappa=\frac{\tan \varphi_{t h r e s h} \cdot g}{v} \\
\dot{\theta}_{m c y} & =\sin \varphi_{t h r e s h} \cdot \dot{\psi}_{g l o b} \\
\dot{\psi}_{m c y} & =\cos \varphi_{t h r e s h} \cdot \dot{\psi}_{g l o b} \\
\dot{\omega}_{\text {thresh }, m c y} & =\dot{\theta}_{m c y}+\dot{\psi}_{m c y}
\end{aligned}
$$

In the above equations, the yaw rate $\dot{\psi}_{g l o b}$ describes the rotation rate about a global inertial system whereas $\dot{\theta}_{m c y}$ and $\dot{\psi}_{m c y}$ describe the rotation rates within the motorcycle coordinate system.

Only one threshold value for the desired roll angle and the measured (GPS) speed is needed within the equations $4-6$. With a maximum threshold roll angle of $\varphi_{\text {thresh }}=3^{\circ}$, the curve for the sum of the rotation rates follows in Figure 6.

Combining the rotation rate criterion with the speed and acceleration criterion, maneuvers for running straight without accelerating or decelerating can be selected. Figure 7 shows the result of the data point selection.

The calculation of the pitch and roll angle from the triaxial acceleration data follows the convention of a rotation sequence $X-Y-Z$, which corresponds to the extrinsic rotation sequence for the $Z-Y-X$ sequence prescribed in DIN 8855 [27]. The following equations apply to the calculation of the smartphone pitch and roll angles:

$$
\begin{aligned}
& \varphi_{S P}=\operatorname{atan}\left(\frac{a_{y}}{a_{z}}\right) \\
& \theta_{S P}=\operatorname{atan}\left(\frac{-a_{x}}{\sqrt{a_{y}^{2}+a_{z}^{2}}}\right)
\end{aligned}
$$

Due to the permitted threshold values for the selection of the data points, a scattered range for the angles of rotation results. The actual rotation angle is determined from the median of the data points, which can be e.g. read graphically or selected automatically from a representation of the cumulative frequency function (CDF) of a data set - see Figure 8.

Step 2 In the second step (see Figure 5), the accelerations and rotation rates are transformed into a horizontal coordinate system in a first transformation with the assumption $\psi_{S P}=0$. This means that the Z-axes of smartphone and motorcycle coordinate system already match, so that the transformed yaw rate matches the yaw rate in the motorcycle coordinate system. 


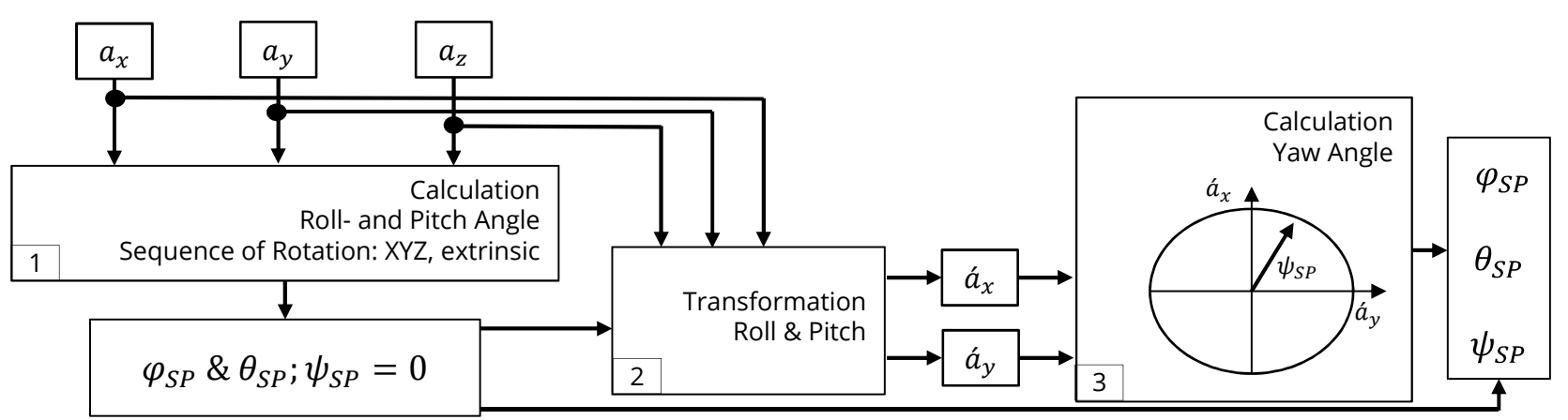

The determination of the rotation angles between the smartphone and the motorcycle coordinate system is divided into three steps. First, the roll and pitch angles are determined from the acceleration due to gravity which is always effective. Then, the inertial measurement data is pre-transformed into the horizonted coordinate system. In the third step, the yaw angle can be determined from the effective acceleration during braking maneuvers when driving straight ahead.

Figure 5. Method for coordinate transformation

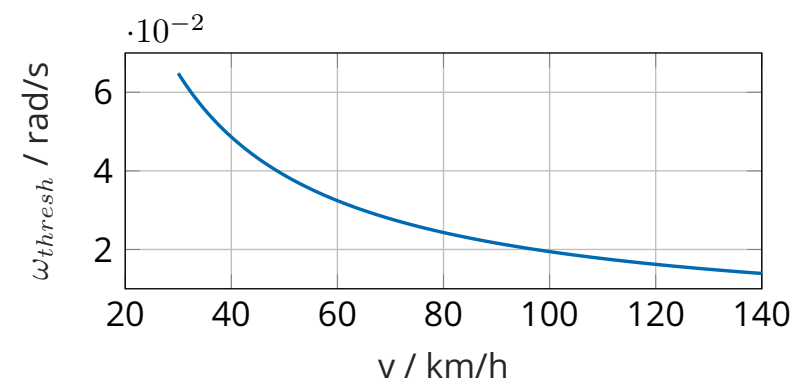

Figure 6. Velocity dependent rotational rate threshold value for running straight; $\varphi_{\text {thresh }}=3^{\circ}$

Step 3 In the third step, the selection of valid data points, which are used to determine the yaw angle, is based on the criterion "braking straight ahead". A straight-ahead ride is determined by comparing the threshold value from Eq. 6 only and the now transformed yaw rate $\dot{\psi}^{\prime}$. To determine sections while braking, the sum of accelerations is formed from the transformed accelerations $a_{x}$ and $a_{y}$. The sign missing due to the squaring of the terms is determined from the filtered time derivative of the GPS velocity $v_{G P S}$. Finally, the data points with a braking acceleration of at least $a_{x, m c y}=2,5 \mathrm{~m} / \mathrm{s}^{2}$ and a minimum speed of $v_{\min }=30 \mathrm{~km} / \mathrm{h}$ are provided for further evaluation. Then, the missing yaw angle can be calculated from the horizonted selected data (Eq. 10):

$$
\psi_{S P}=\operatorname{sign}\left(a_{G P S}\right) \cdot \cos \left(\frac{a_{x}^{\prime}}{\sqrt{a_{x}^{\prime 2}+a_{y}^{\prime 2}}}\right)
$$

Similar to the above procedure, the yaw angle is also defined by the median of the calculated angles $\psi_{S P}$ (see Figure 8).

Data Transformation With the now known angles of rotation of the smartphone to the motorcycle, all inertial raw data are transformed into the motorcycle coordinate system by transformation using the

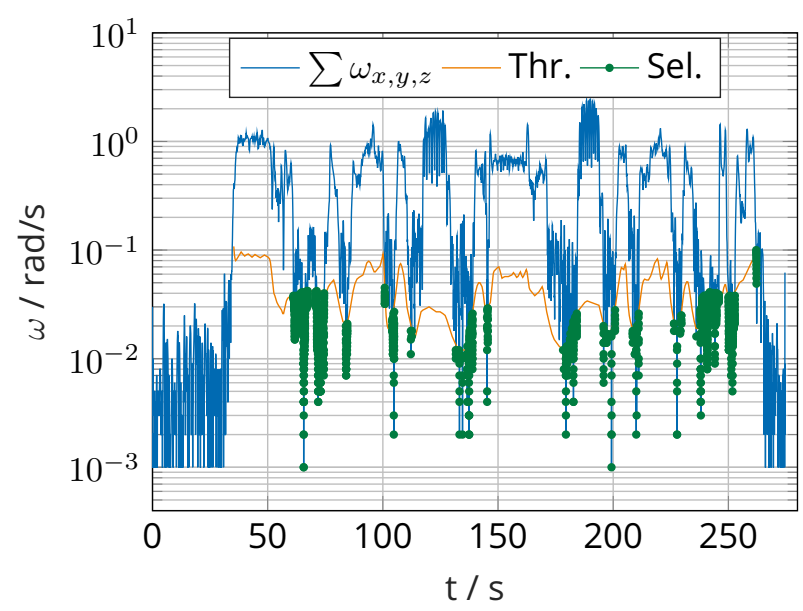

The threshold value from Eq. 7 is applied to the sum of all smartphone rotation rates. The data points from the rotational velocity criterion are then overlaid with the criterion of constant speed. The selected data points (in green) are used for the calculation of the pitch and roll angle of the smartphone.

Figure 7. Selection of datapoints for running straight

transformation matrices $R_{X, Y, Z}$ (Eqs. 11-13) which result in $R_{X Y Z}$ (Eq. 14):

$$
\begin{aligned}
R_{X}= & \left(\begin{array}{ccc}
1 & 0 & 0 \\
0 & \cos \left(\hat{\varphi}_{S P}\right) & -\sin \left(\hat{\varphi}_{S P}\right) \\
0 & \sin \left(\hat{\varphi}_{S P}\right) & \cos \left(\hat{\varphi}_{S P}\right)
\end{array}\right) \\
R_{Y}= & \left(\begin{array}{ccc}
\cos \left(\hat{\theta}_{S P}\right) & 0 & \sin \left(\hat{\theta}_{S P}\right) \\
0 & 1 & 0 \\
-\sin \left(\hat{\theta}_{S P}\right) & 0 & \cos \left(\hat{\theta}_{S P}\right)
\end{array}\right) \\
R_{Z}= & \left(\begin{array}{ccc}
\cos \left(\hat{\psi}_{S P}\right) & -\sin \left(\hat{\psi}_{S P}\right) & 0 \\
\sin \left(\hat{\psi}_{S P}\right) & \cos \left(\hat{\psi}_{S P}\right) & 0 \\
0 & 0 & 1
\end{array}\right) \\
R_{X Y Z}= & R_{X} \times R_{Y} \times R_{Z}
\end{aligned}
$$

After the transformation all inertial data are available in the motorcycle coordinate system and are 


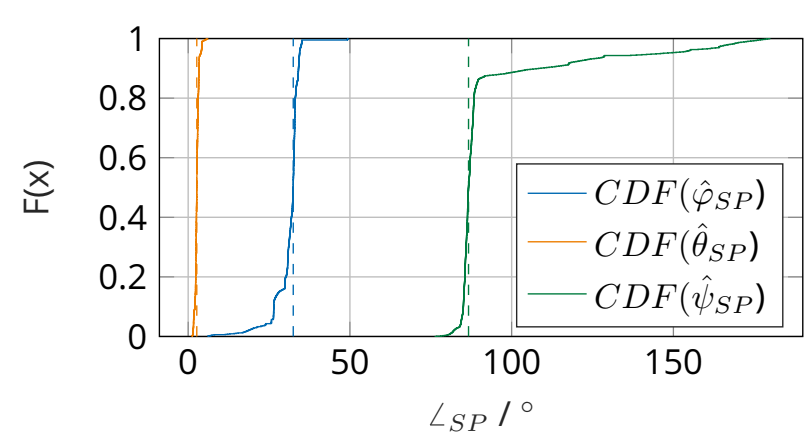

Plot of the CDF and the selected values for smartphone roll angle $\hat{\varphi}_{S P}=2.7^{\circ}$ and pitch angle $\hat{\theta}_{S P}=32.5^{\circ}$ (additionally the yaw angle $\hat{\psi}_{S P}=86.7^{\circ}$, see below)

Figure 8. Selection of transformation angle values

stored next to the raw data in the measurement dataset.

\subsection{Data Filtering}

The recorded raw measurement data from the smartphone on the motorcycle is characterized by a high proportion of unwanted vibration components, which are due on the one hand to the vehicle's own vibrations of the combustion engine drive train and on the other hand to external excitation such as road or air excitation. These vibration components must be filtered out for data evaluation to investigate the actual behavior of the riders. In comparison to the driving dynamics of the rider and the whole vehicle, these additional influences are higher-frequency vibration components. This characteristic makes a low-pass filter suitable for removing these components.

According to [31], the bandwidth of the roll dynamics is about $3 \mathrm{~Hz}$. The rotational rates are therefore filtered with a frequency lying above that value. In [24], the cutoff frequency is $1 \mathrm{~Hz}$ for accelerations. Following cut-off frequencies result for the various data channels, see table 1 :

Table 1. Lowpass Filter frequencies

\begin{tabular}{c|r} 
parameter & $f_{c} / \mathrm{Hz}$ \\
\hline accelerations & $1 \mathrm{~Hz}$ \\
rotational rates & $5 \mathrm{~Hz}$ \\
forward velocity & $0,3 \mathrm{~Hz}$
\end{tabular}

Specifically, a low-pass filter with Butterworth characteristics is used. Besides, the offline evaluation includes forward and backward filtering, since no real-time calculation is necessary and the phase shift between channels that would otherwise result can be eliminated. Finally, Figure 9 shows an example of the post-processing of a longitudinal acceleration signal $a_{x}$ with raw data and filtered data.

In this example, the cause of the high-frequency disturbance is the fixture of the smartphone which has an eigenfrequency of about $18 \mathrm{~Hz}$. Through fix- ing the smartphone to fairing parts or the rear seat, these disturbances can be eliminated partially "mechanically" while data acquisition (see Figures 11 and 17).

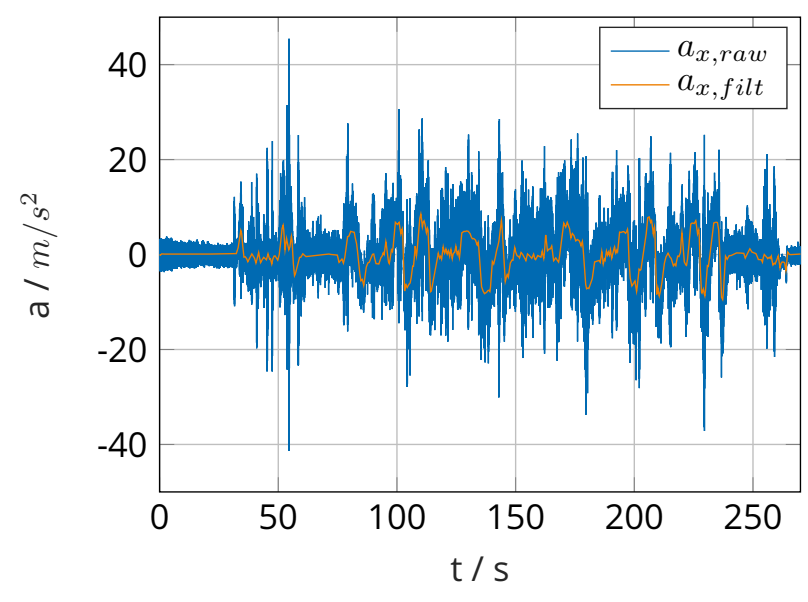

The high-frequency disturbance components of the signal can be effectively filtered out with the cut-off frequency used, resulting in a smoothed and physically plausible signal which is used for further investigations.

Figure 9. Example for data filtering

\subsection{Roll Angle Estimation}

Due to the recording of measurement data using a smartphone, there are some limitations for the roll angle calculation. Thus, a method has to be implemented providing the most precise calculation results for the roll angle despite negative properties of the data signals. Since a calculation of the roll angle by GPS position or GPS speed does not yield sufficiently accurate results (low sampling rate \& accuracy), the calculation of the roll angle should preferably be based on the measurement data of the inertial sensor system of the smartphone rather than relying on GPS data only.

To minimize the number of necessary calculation data, the additional roll angle is calculated directly from the available measurement data. The lateral $a_{y, m c y}$ and vertical $a_{z, m c y}$ acceleration acting in the motorcycle coordinate system can be used for this purpose, meaning that e.g. measurement of the tire width or estimation of the additional roll angle due to tire width is not necessary. Therefore, we propose the methodology for roll angle estimation depicted in Figure 10.

Switching between the stationary roll angle channels is introduced because accelerometers that are not calibrated exactly can cause deviations of the roll angle around the upright position. The effort of a sensor calibration (e.g. [40]) was deliberately omitted, because it might not be possible to perform it reliably in case of a distribution of the smartphone app over a larger rider collective. For small roll angles predicted in the Kalman filter $\varphi<10^{\circ}$, the roll angle is calculated from the longitudinal speed and 


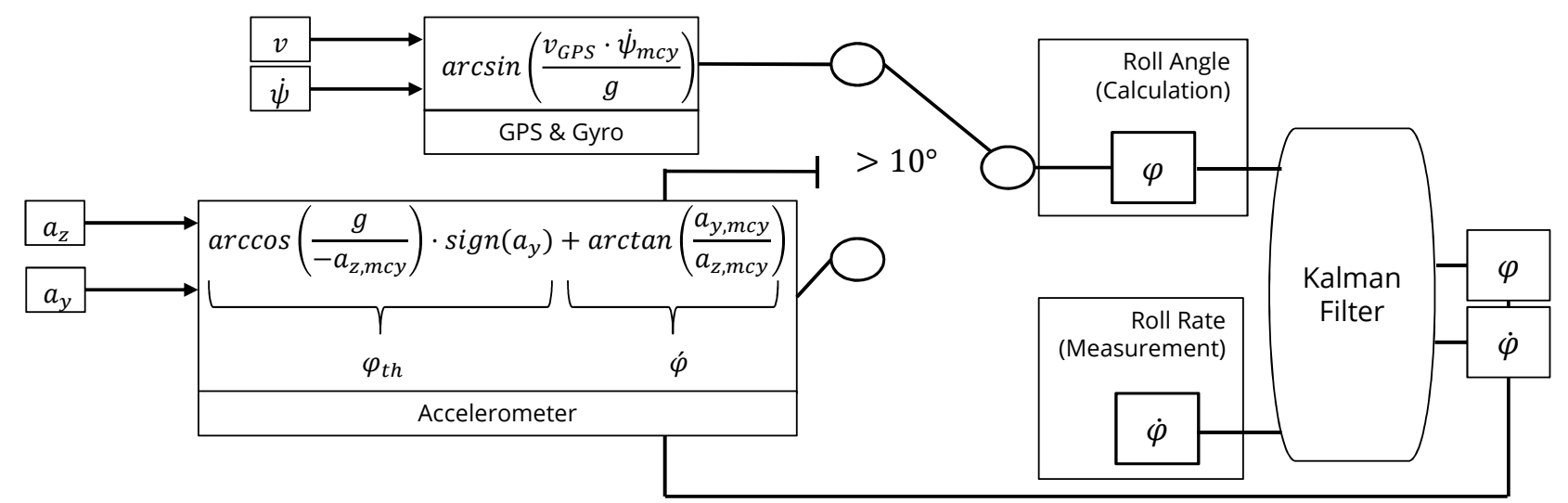

Schematic structure of the lean angle estimation algorithm used with a linear Kalman filter. In the Kalman filter, three measurement data channels are merged. The actual roll angle is supplied as a calculated channel, whereby a distinction is made between an approximate straight-ahead travel and curve travel by switching at a limit roll angle of $\varphi_{\text {lim }}=10^{\circ}$. The calculated stationary lean angle is then fused with the measured roll rate $\dot{\varphi}$ by a linear Kalman Filter.

Figure 10. Algorithm for roll angle estimation

yaw rate (see Eq. 15). Especially on straight sections of the track, the GPS speed is sufficiently accurate so that the longitudinal speed can be used here.

$$
\varphi_{m e a s, G P S}=\operatorname{asin}\left(\frac{v_{G P S} \cdot \dot{\psi}_{m c y}}{g}\right)
$$

For large roll angles $a b s(\varphi)>10^{\circ}$ the Kalman filter switches to the calculated signal from the acceleration sensors. Since the theoretical roll angle is calculated purely from the vertical acceleration in the motorcycle plane, this value is not signed, which is why the sign is taken from the lateral acceleration (Eq. 16). The additional roll angle due to tire width is calculated from the quotient of the lateral acceleration and vertical acceleration (Eq. 17). The sum (Eq. 18) of the two calculated values gives the stationary roll angle.

$$
\begin{aligned}
\varphi_{a z} & =\operatorname{sign}\left(a_{y, m c y}\right) \cdot \operatorname{acos}\left(\frac{g}{-a_{z, m c y}}\right) \\
\varphi_{a y} & =\operatorname{atan}\left(\frac{a_{y, m c y}}{a_{z, m c y}}\right) \\
\varphi_{\text {meas }, a c c} & =\varphi_{a z}+\varphi_{a y}
\end{aligned}
$$

Since the calculated roll angle channels are only valid for stationary cornering and a pure integration of the roll rate does not deliver a sufficient result (see [32]), the suitability of the algorithm for dynamic driving maneuvers is achieved by fusion with the roll rate with the use of a linear Kalman Filter (for details, see e.g. [41]).

The following state vector is used where $\varphi_{\text {meas }}$ refers to the calculated roll angles in Eqs. 15 and 18. The roll rate $\dot{\varphi}$ is the actually measured and transformed value from the smartphone $\omega_{x, m c y}$.

$$
x=\left[\varphi_{\text {meas }}, \dot{\varphi}\right]^{T}
$$

\subsection{Course Angle Calculation}

The course angle signal calculated in the smartphone with the GPS positions is already available in the recorded measurement data record through the GPS API; short for Application Programming Interface. However, after evaluating several test drives, this signal does not meet the requirements for a more precise evaluation. The inaccuracy is due to sporadic outages and the time resolution of the measurement signal ( $1 \mathrm{~Hz}$ sampling rate). As a consequence, a function is integrated into the postprocessing environment which allows the calculation of the course angle from the recorded GPS positions. For the calculation, it should be noted that the GPS coordinate system is not Cartesian and therefore the points are first transferred to a Cartesian system via UTM (Universal Transverse Mercator) transformation (see e.g. [42]). Using this method, the earth's surface is in parts transformed into a Cartesian coordinate system. Equation 20 shows the formula on which the calculation with the transformed coordinates is based. The indices " 2 " and " 1 " in this context denote successive measured data points where the calculation variables $x$ and $y$ originate from the UTM transformation.

$$
\cos (\psi)=\frac{y_{2}-y_{1}}{\sqrt{\left(x_{2}-x_{1}\right)^{2}+\left(y_{2}-y_{1}\right)^{2}}}
$$

The result of the calculated course angle provides the angle between the direction of travel and the north direction. As a result, maximum values of $\pm 180^{\circ}$ are thus achieved, which must be taken into account, for example, when calculating the change in course angle of curves.

\subsection{Curvature and Curve Cegmentation}

For the determination of useful curve segments, the respective curve radius is calculated from the 
recorded driving data which can then be used for segmentation of the data.

Curvature The curve radius can be calculated with different data, however, the sometimes faulty (GPS) speed is usually included in the calculation term. The following formula is used to ensure that this speed is not included in the calculation as far as possible:

$$
R_{c}=\frac{v \cdot \cos (\varphi)}{\dot{\psi}_{m c y}}=\frac{1}{\kappa}
$$

The resulting signal is saved for further inquiries as the inverse curve radius (curvature $\kappa$ ).

Curve segmentation To evaluate curve features like the maximum driven roll angle from a statistical point of view, automated data preparation and segmentation has to be carried out to evaluate individual events. For automated segmentation, the curvature is searched for sign changes. A segment between two sign changes is defined as a curve if the course angle of the motorcycle within the segment changes by more than $\psi_{\text {thresh }}=10^{\circ}$. Gorges et al. [43] set this value to $\psi_{\text {thresh }}=60^{\circ}$ which is more restrictively sorting out curve segments. Their curve detection algorithm is not based on a course angle directly, but they calculate the course angle difference from the timespan, velocity and mean curve radius of the curve.

\section{Measurement Chain Verification}

After developing the methods to measure motorcycle dynamics, they are verified by comparing the smartphone measurement chain to a high precision inertial measurement unit.

\subsection{Inertial Measurement Unit (IMU)}

The $A_{D M A^{5}} G$ inertial measuring system from GeneSys [28] is used for comparative measurements in Dresden and Darmstadt. ADMA G is a high-precision measuring system that was specially developed for vehicle dynamics measurements in the automotive sector to determine acceleration, speed, position and rotation rates in all three spatial directions while driving. A fusion algorithm (EKF) calculates speed, position and location in space from the raw data and compensates measurement inaccuracies of the individual sensors. In addition, the drift behavior is compensated by using a GPS receiver, resulting in a highly precise measurement signal. The size of the ADMA is very compact so that it can be easily attached to the motorcycle.

The system has already been used in other studies for measuring the driving dynamics and roll angle of motorcycles, so that it also appears suitable for validating the smartphone. Schlipsing et

\footnotetext{
${ }^{5}$ Automotive Dynamic Motion Analyzer
}

al. [38] used the ADMA system for reference measurements of a low-cost IMU as well as for roll angle calculation from camera data.

\subsection{Test Vehicle}

A BMW R1200 GS Adventure (K51) motorcycle is used for the comparison and validation measurements. Figure 11 shows the motorcycle on the test track with the measuring systems to be compared (Smartphone and ADMA).

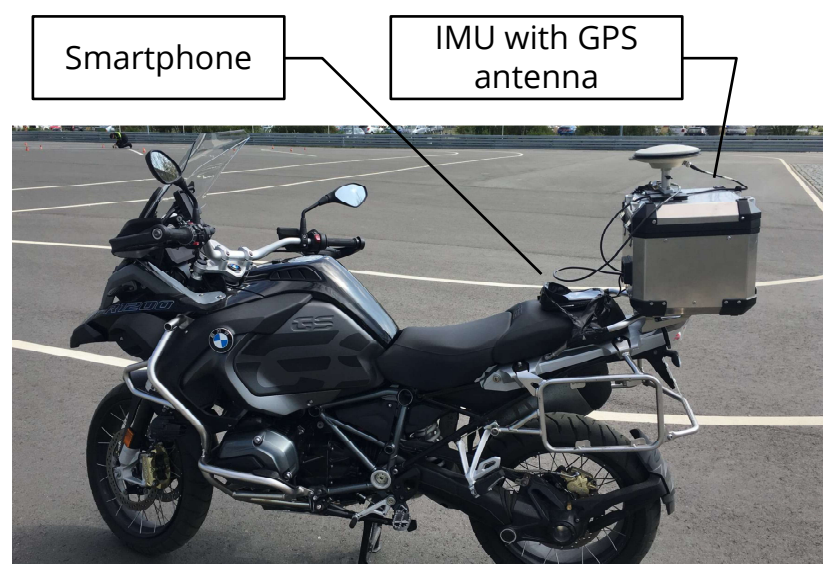

Figure 11. Test vehicle equipped with two data acquisition systems

The IMU is fixed within the topcase for testing purposes and connected to the on-board power supply. In addition, the GPS antenna is mounted on the lid of the topcase. For data logging of the IMU-measurement-data, a laptop is used, which is carried in the backpack of the rider. For the validation ride on the test tracks the smartphone is either fixed on the pillion seat utilizing adhesive tape and a cover, on a professional fixture system (SW-Motech ${ }^{6}$ ) X-Grip or directly on fairing parts. It should be noted that both measuring systems must be installed on the vehicle so that they are fixed to the body.

\subsection{Test Tracks}

Two test tracks are used for the validation phase. The first one is an airfield near Darmstadt, see Figure 12

For the design of the Dresden test track, it was important that the majority of the test track should run on rural roads with many bends, as there is a particular accumulation of fatal accidents. The route selection is therefore also based on sections of the "Accident Atlas" of the German statistical offices of the federal and state governments. [44]

As a general condition for the test track, the area of the Technische Universität Dresden will be defined as start and finish point. For the detailed planning of the course, left and right curves should preferably occur in equal parts. At the same time,

\footnotetext{
${ }^{6}$ SW-MOTECH GmbH \& Co. KG, Rauschenberg, Germany
} 


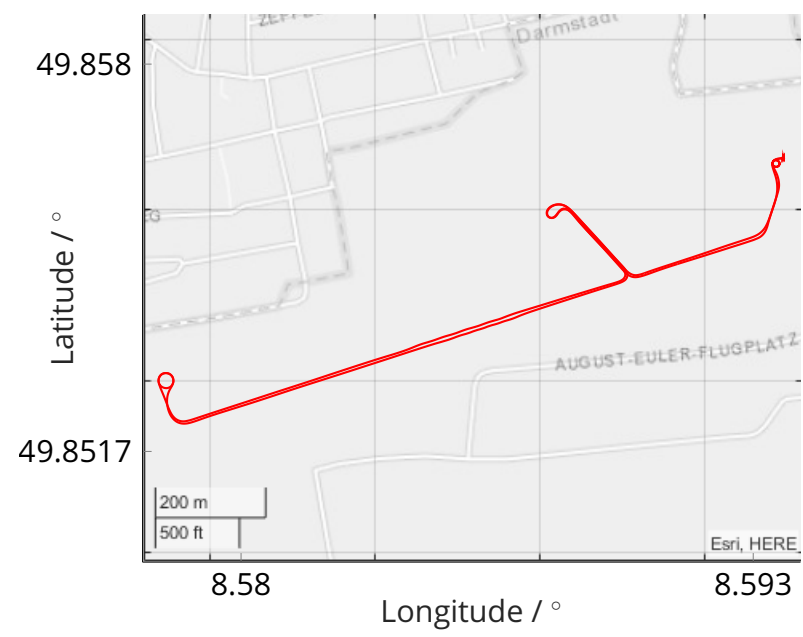

The Darmstadt test track is assembled of different sections at the August-Euler airfield nearby Darmstadt. With a total length of $4 \mathrm{~km}$, it is absolved in about 5 minutes. (C)OpenStreetMap contributors

Figure 12. Test track in Darmstadt

curves with as different radii as possible should be covered in the course of the route. Finally, this results in the route shown in Figure 13.

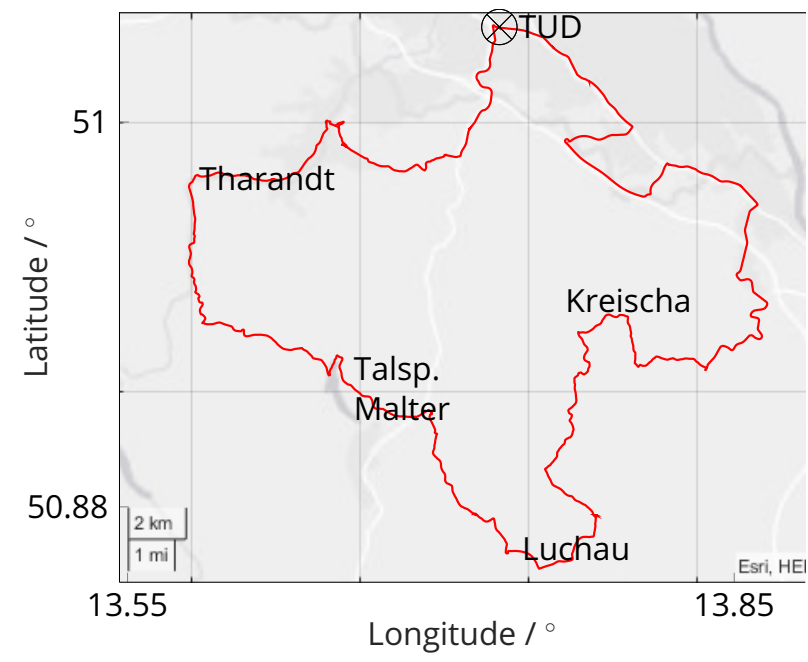

The Dresden test track has a total length of $84 \mathrm{~km}$ and a journey time of about one hour and 45 minutes. A significant part of the track has to be completed in the nearby Erzgebirge. (c) OpenStreetMap contributors

Figure 13. Test track in Dresden

\subsection{Test Setup}

The test tracks in Darmstadt (DA) and Dresden (DD) are used in different combinations with the two Data Acquisition (DAQ) systems, see Table 2. IMU and Smartphone data are compared for the Darmstadt (DA) proving ground and the Dresden test track (DD 1). Then, further studies are carried out without the IMU (DD 2)
Table 2. Data acquisition and test track configurations

\begin{tabular}{c|ccc} 
DAQ & DA & DD 1 & DD 2 \\
\hline \begin{tabular}{c|c} 
Smartphone \\
IMU
\end{tabular} & $\bullet$ & $\bullet$ & $\bullet$ \\
$\bullet$
\end{tabular}

\subsection{Method Verification}

To verify and validate the proposed method, we first give an overview of the basic motorcycle roll dynamic parameters. Then, the data quality delivered by our additional calculations or estimations is investigated. Since the coordinate transformation algorithm is an important part of the whole measurement chain, we deliver a practical example for the reproducibility of our whole measurement chain comparing three smartphones mounted on one motorcycle in different orientations. Finally, the data quality delivered by the most important sensors is investigated.

Overview In the same manner, as the authors do in [36], we depict the speed, roll and yaw rates, roll angle and roll angle error graphs within Figure 14. Concentrating on the roll angle plot $\varphi$, the traces show an excellent correlation between the ground - truth - data from the IMU and the smartphone measurement chain. The difference plot $\Delta \varphi$ on the bottom reveals that typical deviations while stationary cornering or riding straight are at max. $3^{\circ}$ and while rolling dynamically, the deviations are below $6^{\circ}$. Despite the data are synchronised by their GPS - timestamps, the biggest deviation source can be seen as the time lag between the systems. Especially due to the roll rate being over twice as fast as in [36], bigger roll angle deviations would be expected, but do not occur. The RMSE value for $\varphi$ is $1.8^{\circ}$ for this example dataset and typical values lie around $2^{\circ}$. Switching between the calculated roll angle channels to be fed into the Kalman Filter at $\varphi=10^{\circ}$ has no negative effect on the estimation.

Curvature Small curve radii $(\kappa>0.1)$ are calculated inaccurately from the measurement data due to error propagation, but larger curve radii are almost identical to the IMU result, see Figure 15.

The biggest error here is due to the low sampling rate of the GPS. Therefore, a function is integrated into the post-processing algorithm that detects implausible values of the inverse curve radius and sets the inverse curve radius to 0 for those cases. The threshold value is $\kappa_{\text {thresh }}=0.25 \frac{1}{m}$, which corresponds to a curve radius of $R_{C} \leq 4 m$ and less. Curves with even smaller radii are not relevant. Despite the absence of an unreadable error plot, we can state that the curve radius estimation error for the stationary curves around $45 \mathrm{~s}$ is about $3 \mathrm{~m}$ and from $150 \mathrm{~s}$ about $4 \mathrm{~m}$. These errors are acceptable and far better than an inverse curve radius calculated from GPS data only. 

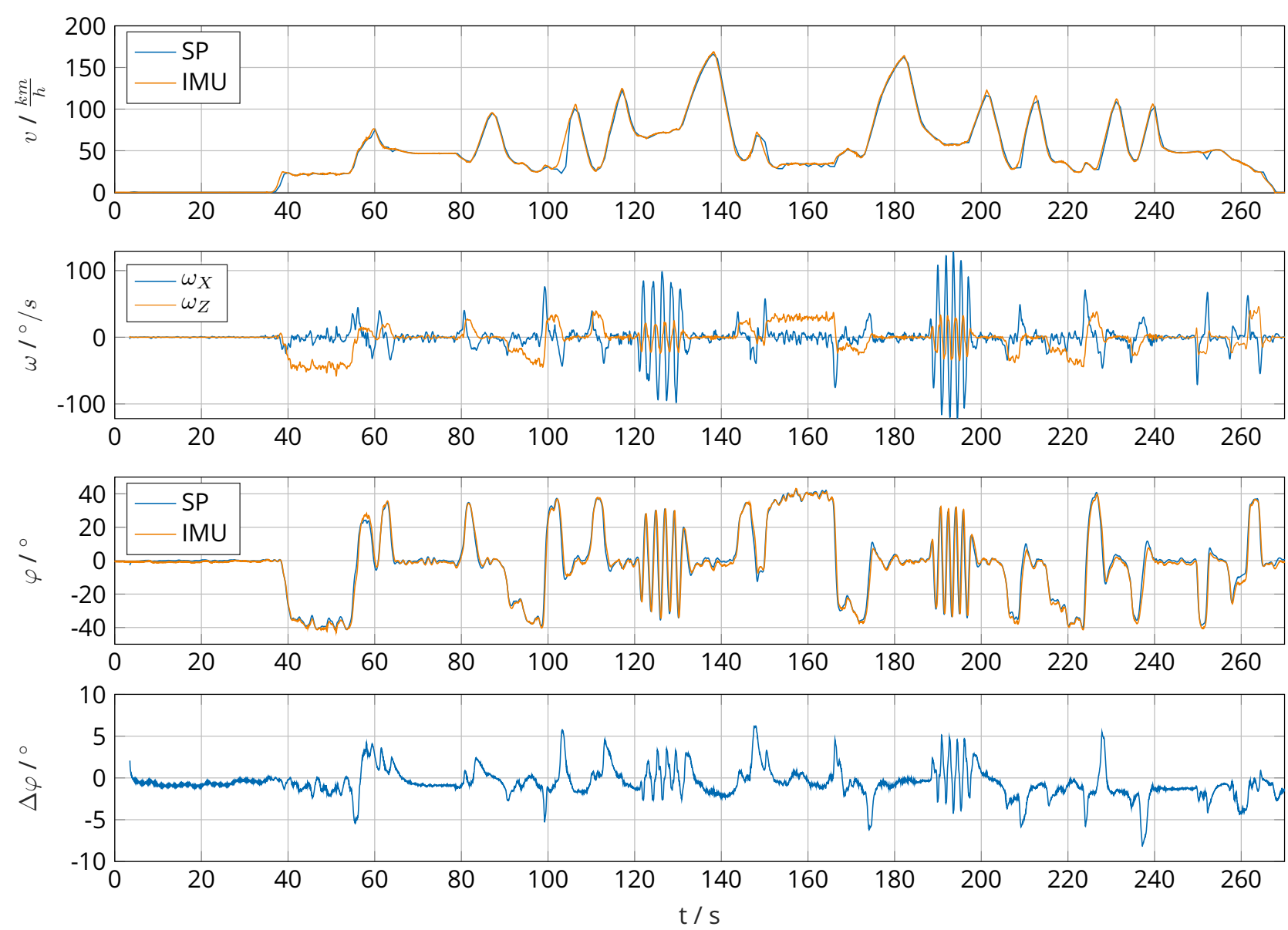

Figure 14. Typical Motorcycle Dynamics Parameters: Speed, Rotational Rates and Roll Angle.

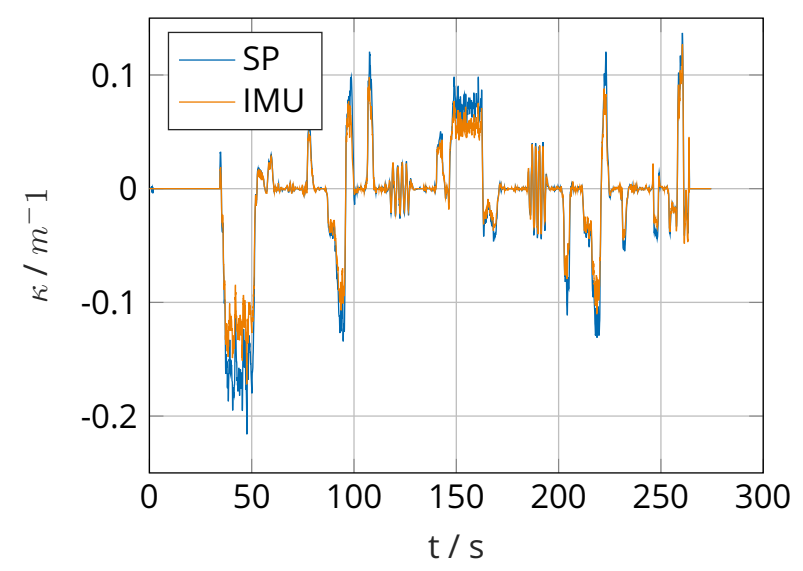

Figure 15. Comparison of the curvature calculation

Segmentation Figure 16 on the right shows the graph of the curve $\kappa$. As there are left and right curves within the driven route (see GPS map on the left), there are positive and negative values for $\kappa$.

When the segmentation algorithm from section 2.6 is applied to the data, the curve segments are effectively separated from the straight or slalom sections. Each colour-highlighted section corresponds to one curve segment which can then be used for further feature extraction.
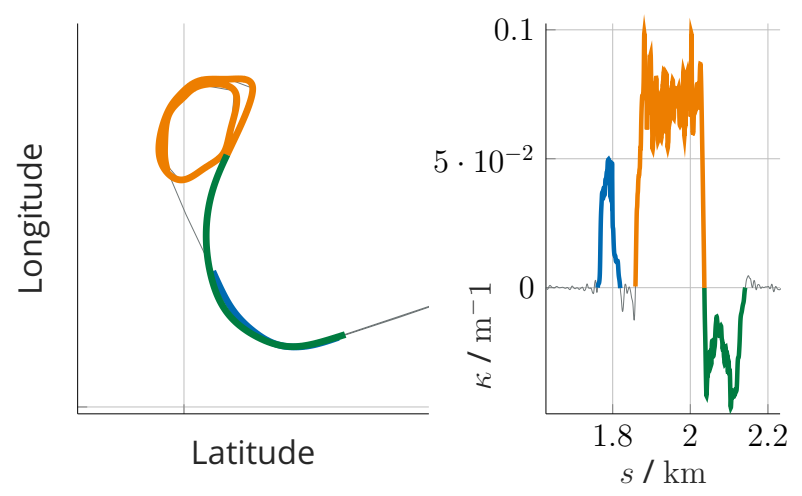

Figure 16. Curve segmentation

Coordinate Transformation The goal of the coordinate transformation algorithm is to enable reproducible measurement values. The authors, therefore, present a comparison of the calculated roll angle of three differently mounted smartphones with parallel data acquisition of the IMU. The results shown before in Figure 14 are the ones from the second smartphone in Figure 17 which shows the test configuration for the coordinate transformation test.

To be able to compare the datasets in Figure 18, the time offset between the datasets is calculated via the logged GPS - times and their known origins. 


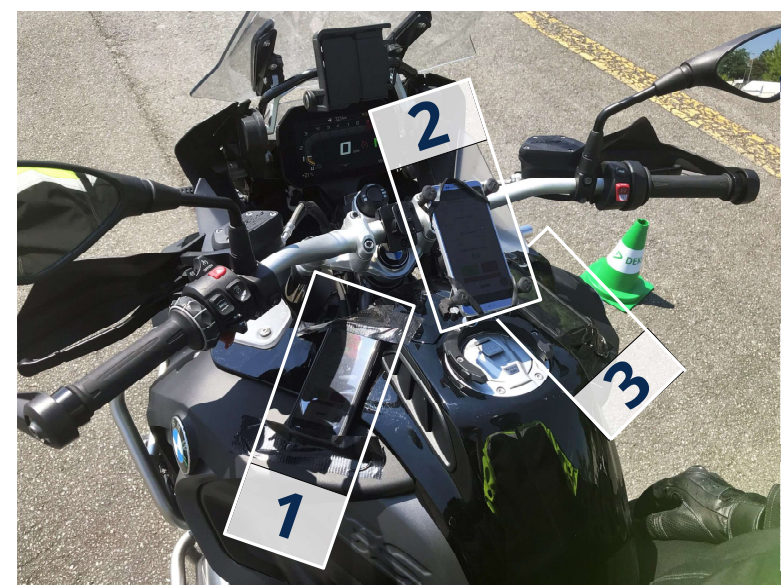

Figure 17. Three smartphones mounted on the test motorcycle in different orientations

It can be stated that the result of the transformation algorithm provides an excellent result as the roll angle estimations with the transformed inertial data still show a good correlation with the high - precision IMU for different orientations. The comparison between the smartphones shows that typical deviations while stationary cornering are below $3^{\circ}$. The largest errors occur during dynamic rolling with max. $9^{\circ}$. RMSE values are below $2.2^{\circ}$.

Table 3. Transformation angles for the test configuration

\begin{tabular}{c|c|c|c}
$\begin{array}{c}\# \\
\text { type }\end{array}$ & $\begin{array}{c}1 \\
\text { Huawei P20 lite }\end{array}$ & $\begin{array}{c}2 \\
\text { LG G6 }\end{array}$ & $\begin{array}{c}3 \\
\text { LG G7 fit }\end{array}$ \\
\hline$\varphi_{S P}$ & $19.6^{\circ}$ & $3.6^{\circ}$ & $2.7^{\circ}$ \\
$\theta_{S P}$ & $15.8^{\circ}$ & $18.6^{\circ}$ & $32.5^{\circ}$ \\
$\psi_{S P}$ & $38.7^{\circ}$ & $105.4^{\circ}$ & $86.7^{\circ}$
\end{tabular}

Despite there are no real angle measurements to compare the smartphone orientation angles to, we want to give numbers as "rule of thumb" for the estimation of the orientation angles. Based on standard deviations, the pitch and roll angles usually are within an interval of $\sigma_{\varphi, \theta}= \pm 5^{\circ}$ and the yaw angle can be estimated by $\sigma_{\psi}= \pm 10^{\circ}$. However, by picking the median of the estimated angles, the method becomes rather robust to outliers in the estimated angles.

Rotational Rates As the rotational rates form the basis of the calculations and therefore could be an error source for the following methods, they should also be assessed regarding their quality. However, they can only be compared with the IMU data after being transformed into the motorcycle coordinate system. The roll and yaw rate are further investigated.

A roll rate time plot (see Figure 19) shows that the graphs overlay so that they cannot be distinguished from each other. An error plot at the bottom shows that the three smartphones deliver a maximum roll
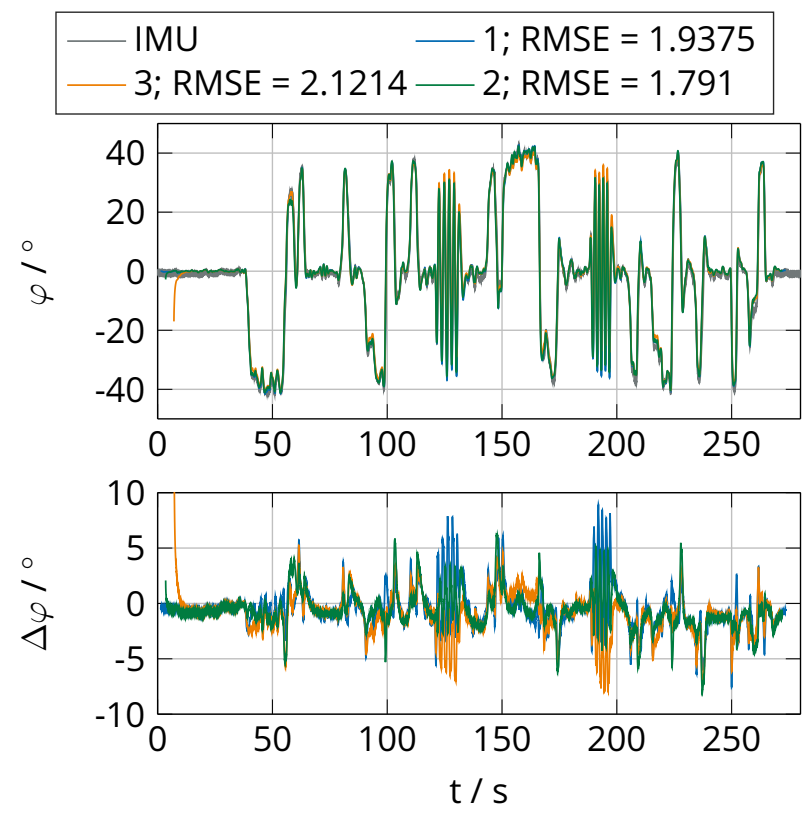

Figure 18. Roll Angle Error Plot / Transformation method test

rate error of $6^{\circ} / \mathrm{s}$ for absolute values of about $130^{\circ} / \mathrm{s}$. That results in a relative roll rate error of under $5 \%$.
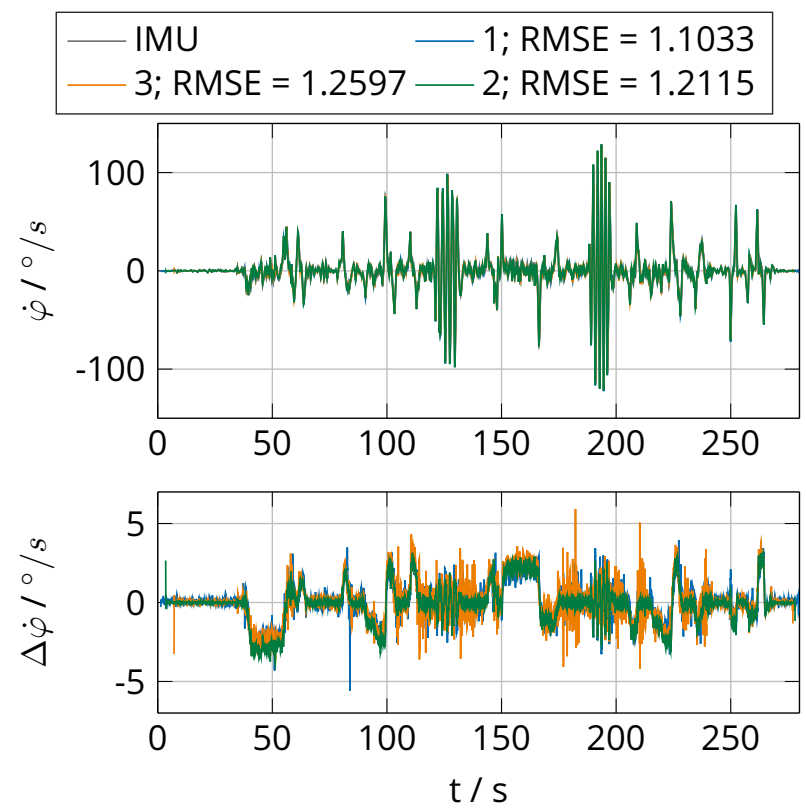

Figure 19. Roll Rate Error Plot

The yaw rate time plot (see Figure 20) reveals nearly the same overlaying quality compared to the roll rate graphs. While the maximum yaw rate value is nearly $60^{\circ} / \mathrm{s}$, the maximum error is $7^{\circ} / \mathrm{s}$ which results in a relative yaw rate error of under $12 \%$.

While the yaw rate does not show a systematic error correlation, the roll rate error is crosscorrelated to the estimated roll angle $\varphi$ with $R=0.8$. The reason for this correlated deviation could be an angle estimation problem within the coordinate transformation algorithm. Looking also into the pitch rate error data which is not depicted here it 

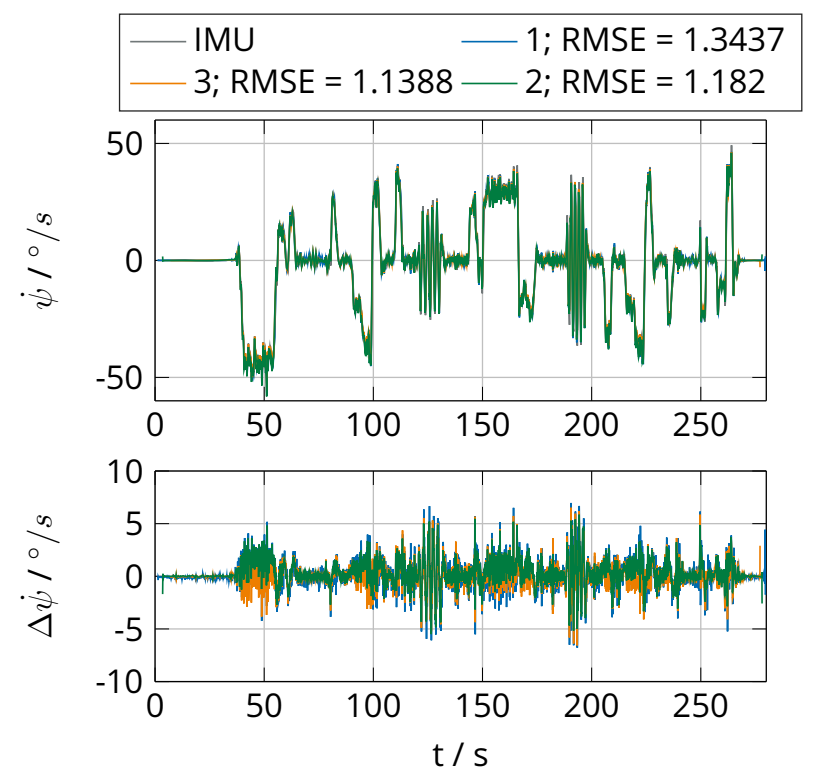

Figure 20. Yaw Rate Error Plot

becomes clear that this is also cross-correlated to the roll angle, but only with $R=40 \%$. From the motorcycle dynamics point of view, this means that the problem can be located to the yaw angle estimation of the smartphone rotation. If the yaw angle estimation $\hat{\psi}_{S P}$ alone is incorrect, the yaw rate will be uncorrelated to the roll angle, but the pitch and roll rate will be affected by the estimation error so that the rotation rates are "crosstalking" into each other because of their misaligned axes. The results for the rotational rate sensors are very encouraging. Further studies should include an enhancement of the smartphone yaw angle estimation.

Longitudinal Acceleration \& Velocity Regarding the GPS velocity, in Figure 21 the data channels from the three smartphones are shown. First of all, the rough peaks from smartphone (3) (orange) can be seen. The main reason is that the additional rugged aluminum cover restricts the GPS receiver so that the quality is worse compared to the other phones. The smartphones (1) and (2) without a cover deliver better quality, but still with a maximum error of up to $31 \mathrm{~km} / \mathrm{h}$. These results are congruent with Neale et al. [45] whereby it may be stated that the low sample rate of $1 \mathrm{~Hz}$ is not suitable to follow "hard" accelerating motorcycles, but riding stationary, errors are small and typically below $5 \mathrm{~km} / \mathrm{h}$.

The transformed longitudinal acceleration recorded by the internal sensor is acquired with a higher sample rate compared to the GPS receiver. In Figure 22, the IMU and smartphone longitudinal accelerations are shown. The IMU acceleration is given in a horizonted coordinate system whereas the smartphone accelerations are left in the motorcycle coordinate system as the smartphone (and motorcycle) pitch angle is unknown for now.
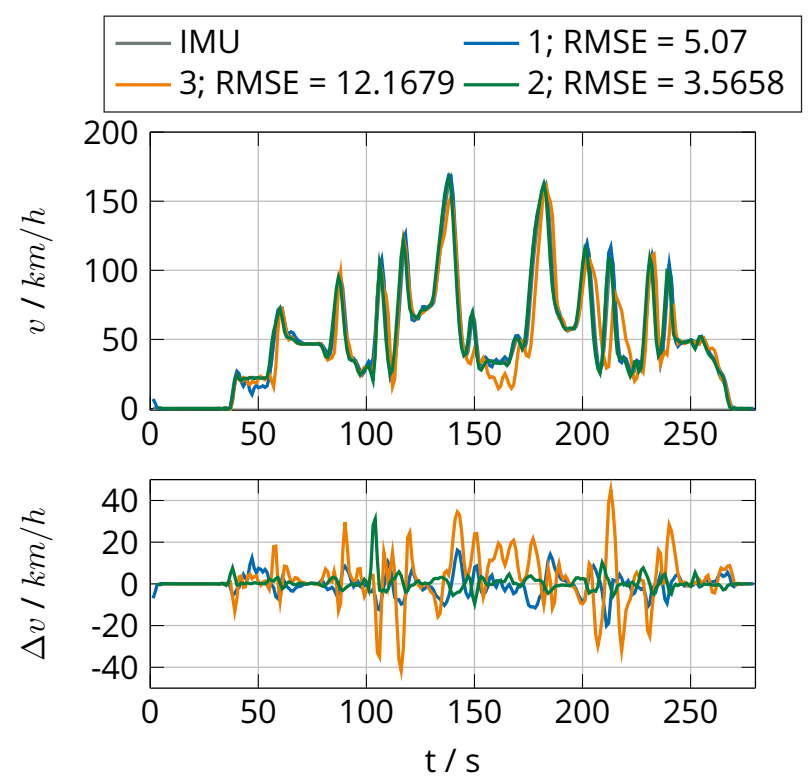

Figure 21. Velocity Error Plot

The maximum error here is $1.5 \mathrm{~m} / \mathrm{s}^{2}$ and occurs while stationary cornering. Even when accelerating or decelerating substantially $\left(\left|a_{x}\right| \approx 8 m / s^{2}\right)$, errors are below $1 \mathrm{~m} / \mathrm{s}^{2}$. The RMSE values for the whole test maneuver are closely around $0.4 \mathrm{~m} / \mathrm{s}^{2}$.

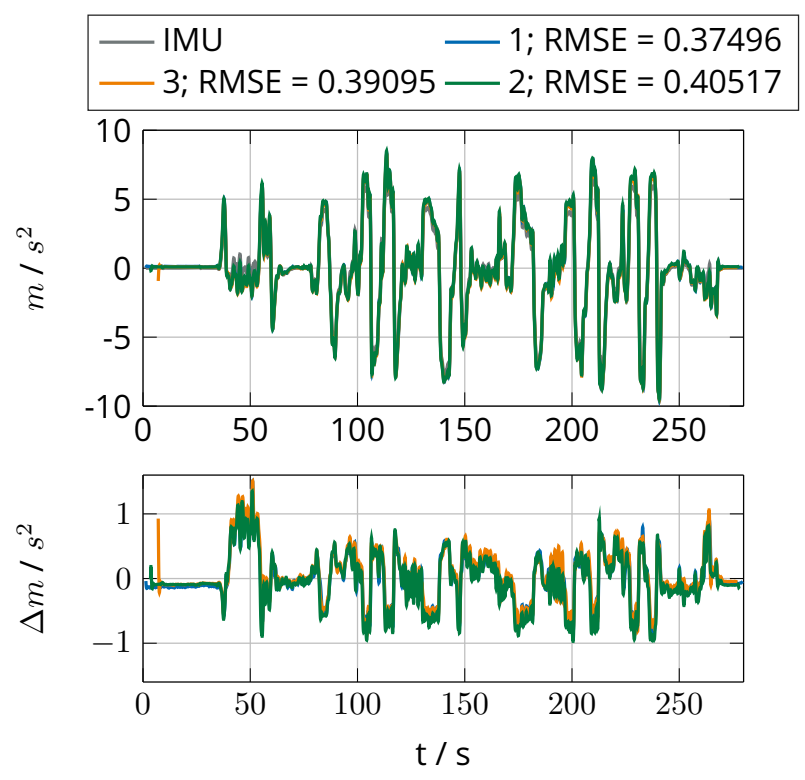

Figure 22. Longitudinal Acceleration Error Plot

As the smartphones themselves show nearly identical values, a systematic error source can be identified as the missing pitch angle correction. Thus, the smartphone acceleration sensors may not be blamed as the main error contributors.

Validation on Dresden test track The same investigations as described in the preceding paragraphs were also carried out for the Dresden test track (configuration DD1). Despite some GPS outages in narrow valleys or deep forest, no additional error 
sources or drawbacks in the overall method could be identified.

\subsection{Proband Study}

After the validation of the whole measurement chain, a proband study with 15 participants was carried out. To avoid wrong expectations, we do not want to present the detailed findings of the study, because all the data collected by us was fused with a dataset from another measurement campaign and presented by Pless et al. [46] which can be understood as an accompanying paper to this work. After fusing the datasets, they look further into the aggregated data and provide valuable findings for future motorcycle safety research.

In our part of the study, different private test vehicles were involved and because of that, it was not possible to develop a uniform holder for the smartphone that is suitable for all motorcycles. Therefore, the smartphone is installed in a simple, quickly exchangeable solution with adhesive tape directly on the seat behind the driver (see Figure 23). From the past measurements in the validation phase, it is known that the fixture on the seat causes much fewer vibrations in the smartphone signals.
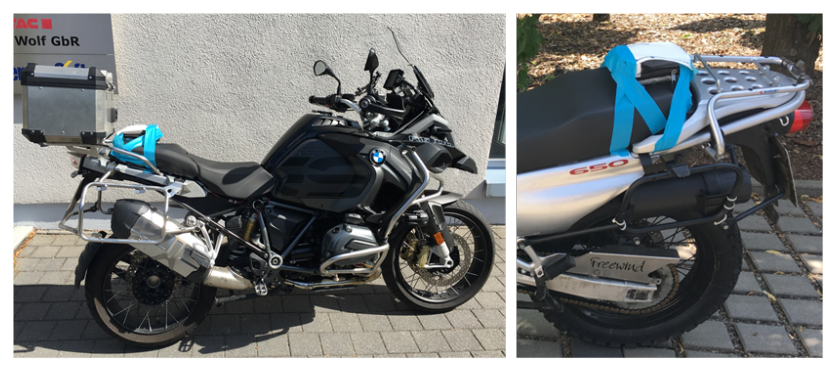

Figure 23. Fixation of the smartphone: Two examples

In Figure 24 the driven lean angles for all curve segments (see section 2.6) and all probands are graphed for left and right curves. It can be seen that the riders nearly use the same lean angles for left and right curves. The graph shows that in $80 \%$ of the observations, for example, the roll angle is up to $25^{\circ}$. Roll angles of more than $30^{\circ}$, which are generally still considered to be harmless, are therefore exceeded in just $10 \%$ of observations.

\section{Results and Discussion}

In the presented paper, we proofed the applicability of a low-cost smartphone-measurement-toolchain for the investigation of driven lean angles of large rider collectives. Thereby, all hardware smartphone sensors showed a good performance in the overall validation. The low sampling rate of the GPS receiver led to some limitations. The power of the developed measurement-toolchain could also be shown in a proband study highlighting that the driven lean angles are under $25^{\circ}$ in $80 \%$ of all observations.

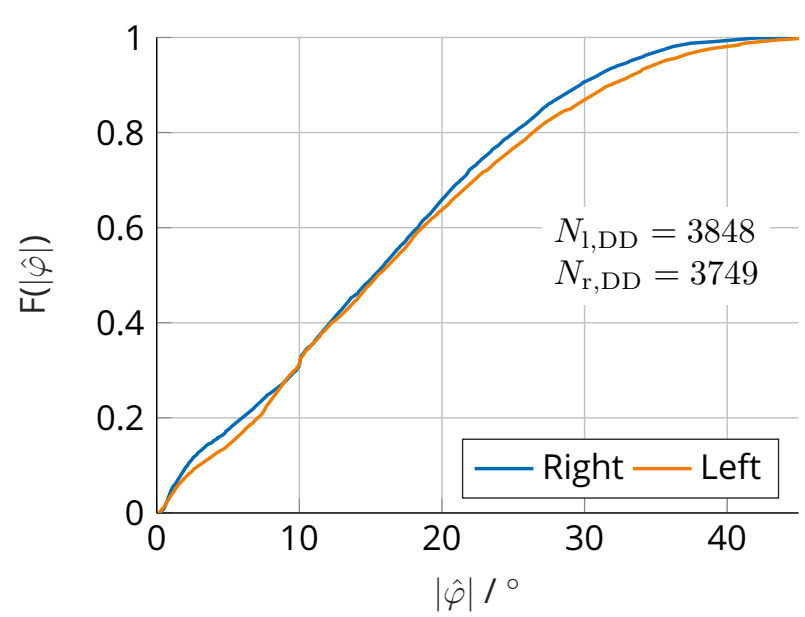

Figure 24. Observed lean angle maxima in left and right turns

The performance of the toolchain is mainly based on splitting it into an on- and offline part. In the online part, the developed Android application "MotoLogger" focuses on high usability and stable data measurement. In the offline part, the implemented MATLAB ${ }^{\circledR}$ processing-toolchain ensures an exact and customizable big data analysis featuring the following highlights: (1) highly robust and motorcycle adapted coordinate transformation algorithm, (2) roll angle estimation using a linear Kalman filter considering the additional roll angle due to tire width and smartphone sensor quality, (3) raw data filtering, (4) course angle calculation and (5) curve segmentation based on curvature being able to analyze a large rider collective.

Future research should especially focus on enhancing GPS sensor performance by fusion algorithms. Apart from that, a pitch angle correction for the longitudinal acceleration signal could also be foreseen in future developments.

In general, researchers could use the developed toolchain for investigating driven lean angles on a large scale. Regarding measurement technology, motorcycles could from now on be equipped with smartphones as they deliver an attractive compromise of price and data quality. From this, specific safety measures for reducing motorcycle accidents could be derived. Furthermore, the toolchain could be the basis for recognizing rider styles (e.g. [47, 48]) and thus adapting the motorcycle's response to each rider individually. Gaining more specific data about the ridden motorcycles, even the estimation of tire width could theoretically be possible. Given the increasing automation of road traffic, the toolchain could be further developed for describing test scenarios to assess automated driving systems $[49,50]$ or evaluate the effectiveness of safety functions for motorcycles [51].

Finally, practitioners could use the gained knowledge to develop a special lean angle training with 
direct lean-angle feedback on the riders' smartphones.

\section{Conclusion}

Driven lean angles of motorcycle rider collectives can be reliably measured by mid-class smartphones, embedded in a holistic measurement toolchain. While the smartphone focuses on collecting raw data, an offline MATLAB ${ }^{\circledR}$ environment realizes the data analysis and lean angle calculation. In general, all smartphone hardware sensors are suitable for dynamic data collection, except the GPS-sensor lacking a sufficient sampling rate. The roll angle estimation algorithm is based on a linear Kalman filter and supported by a robust coordinate transformation algorithm. A big data analysis is ensured by an automatic curve segmentation.

Smartphones as research tools have gained increased attention in the last years. Now, for the first time, a holistic measurement toolchain using smartphones was established and validated to investigate driven lean angles on a large scale. Thus, lean angles of large rider collectives can be investigated in future easily with (1) a low-cost mobile measurement tool and (2) with private motorcycles. We anticipate that our toolchain will help to derive specific safety measures for reducing motorcycle accidents. Moreover, rider styles could be investigated in more detail and a lean angle training with direct feedback could be established.

\section{Acknowledgments}

This report is based on the research project carried out at the request of the Federal Ministry of Transport and Digital Infrastructure, represented by the Federal Highway Research Institute, under research project No. 82.0710/2018. The authors are solely responsible for the content. We want to thank Mr. Luis Köder for supporting the research team during his diploma thesis.

\section{References}

[1] Bräutigam, J., Hellmann, A., Seiniger, P., ed. Sicherheit, Umwelt, Zukunft XII: Tagungsband der 12. Internationalen Motorradkonferenz 2018 = Safety Environment Future : Proceedings of the 12th International Motorcycle Conference. Vol. Nr. 18. ifz-Research Publication Series. Essen, Ruhr: Institut für Zweiradsicherheit, 2018. ISBN: 9783923994236.

[2] Statistisches Bundesamt. Verkehrsunfälle - Kraftrad- und Fahrradunfälle im Straßenverkehr 2018: Artikelnummer: 5462408-18700-4. 2019.

[3] Marcus Bäumer et al. Fahrleistungserhebung 2014 - Inlandsfahrleistung und Unfallrisiko. Berichte der Bundesanstalt für Strassenwesen Verkehrstechnik. Bremen: Fachverlag NW, 2017-08. ISBN: 9783956063374. URL: https://edocs.tib.eu/files/e01fn17/1001674367.pdf.
[4] K. Bauer, S. Peldschus, and S. Schick, eds. Retrospektive Analyse tödlicher Motorradunfälle und Ableitung von Schutzmaßnahmen bei komplexen Bremsmanövern. 2014. URL: https://trid.trb.org/view/1480248.

[5] Bernt Spiegel. Die obere Hälfte des Motorrads: Vom Gebrauch der Werkzeuge als künstliche Organe: [über die Einheit von Fahrer und Maschine. 5. Aufl. Stuttgart: Motorbuch-Verl., 2006. ISBN: 9783613022683.

[6] Martin Winkelbauer, Aggelos Soteropoulos, and Ernst Tomasch. "Unfallursachen bei Motorradunfällen - KFVSicher Leben \#4". In: (2017-06). URL: https : / / www . researchgate.net/publication/320347831.

[7] Christian Hädrich. "Messung der Schräglage von Motorrädern bei Kurvendurchfahrt". Diplomarbeit. Aachen: RWTH Aachen, 2012-09.

[8] Michael Praschl and Julia Schöllbauer. "Evaluation von freiwilligen Motorrad-Fahrtrainings: GZ. BMVIT199.939/0001-IV/IVVS2/2016". In: Im Auftrag des Österreichischen Verkehrssicherheitsfonds Wien (2016-07).

[9] Sebastian Will. "Development of a presence model for driving simulators based on speed perception in a motorcycle riding simulator". Dissertation. Würzburg: JuliusMaximilians-Universität Würzburg, 2017-05-12.

[10] Shane B. McLaughlin, Zachary R. Doerzaph, and Brad R. Cannon. Pilot Study of Instrumentation to Collect Behavioral Data to Identify On-Road Rider Behavior: DOT HS 811442. Apr. 2011.

[11] F. Tenzer. Anzahl der Nutzer von Smartphones in Deutschland bis 2019. Hamburg, 2020-04-01. URL: https:// de. statista.com/statistik/daten/studie/198959/umfrage/ anzahl - der - smartphonenutzer - in - deutschland - seit 2010/ (visited on 07/06/2020).

[12] Marc Schelewsky et al. Smartphones unterstützen die Mobilitätsforschung. Wiesbaden: Springer Fachmedien Wiesbaden, 2014. ISBN: 978-3-658-01847-4. DOI: 10.1007/9783-658-01848-1.

[13] Michael B. del Rosario, Stephen J. Redmond, and Nigel $\mathrm{H}$. Lovell. "Tracking the Evolution of Smartphone Sensing for Monitoring Human Movement". In: Sensors (Basel, Switzerland) 15.8 (2015), pp. 18901-18933. DOI: 10.3390/ s150818901.

[14] Karina Campos et al. Towards a methodology to extract forensics information from the smartphone sensors: Finding evidence: Salinas, Ecuador, October 16-20, 2017. Piscataway, NJ: IEEE, 2017. ISBN: 9781538638958. URL: http:// ieeexplore.ieee.org/servlet/opac?punumber $=8232983$.

[15] Waleed Aleadelat and Khaled Ksaibati. "Estimation of Pavement Serviceability Index Through Android-Based Smartphone Application for Local Roads". In: Transportation Research Record:Journal of the Transportation Research Board 2639.1 (2017), pp. 129-135. ISSN: 0361-1981. DOI: 10.3141/2639-16.

[16] Artis Mednis et al. "Real time pothole detection using Android smartphones with accelerometers". In: 2011 International Conference on Distributed Computing in Sensor Systems and Workshops (DCOSS). 2011, pp. 1-6. DOI: 10.1109/DCOSS.2011.5982206.

[17] Islam Shahidul. "Development of a smartphone application to measure pavement roughness and to identify surface irregularities". Dissertation. Urbana, Illinois: University of Illinois at Urbana-Champaign, 2015.

[18] Rui Araujo et al., eds. Driving coach: A smartphone application to evaluate driving efficient patterns. 2012. DOI: 10.1109/IVS.2012.6232304.

[19] Dongyao Chen et al. "Invisible Sensing of Vehicle Steering with Smartphones". In: Proceedings of the 13th Annual International Conference on Mobile Systems, Applications, and Services - MobiSys '15. Ed. by Gaetano Borriello et al. New York, New York, USA: ACM Press, 2015, pp. 1-13. ISBN: 9781450334945. DOI: 10.1145/2742647.2742659. 
[20] $\mathrm{H}$. Eren et al. "Estimating driving behavior by a smartphone". In: 2012 IEEE Intelligent Vehicles Symposium. IEEE, 3.06.2012 - 07.06.2012, pp. 234-239. ISBN: 978-1-46732118-1. DOI: 10.1109/IVS.2012.6232298.

[21] Yan Wang et al. “Determining Driver Phone Use by Exploiting Smartphone Integrated Sensors". In: IEEE Transactions on Mobile Computing 15.8 (2016), pp. 1965-1981. ISSN: 1536-1233. DOI: 10.1109/TMC.2015.2483501.

[22] Javier Almazan et al., eds. Full auto-calibration of a smartphone on board a vehicle using IMU and GPS embedded sensors. 2013. DOI: 10.1109/IVS.2013.6629658.

[23] L. Hannawald, M. Marschner, and H. Liers. “The Usage Of Smartphones For Recording Accidents And Incidents From The Critical Situation Up To The Post-Crash Phase". In: 22. EVU Conference. Florence.

[24] Tsukasa Kamimura, Tomoya Kitani, and Takashi Watanabe. "A System to Comprehend a Motorcycle's Behavior using the Acceleration and Gyro Sensors on a Smartphone". In: Global Research and Education (2012-08). URL: http://kitanilab.org/publication/data/pdf/4.pdf.

[25] Vittore Cossalter. Motorcycle dynamics. 1. engl. ed. Greendale Wisc.: Race Dynamics, 2002. ISBN: 9780972051408.

[26] Kai Schröter, Raphael Pleß, and Patrick Seiniger. "Vehicle Dynamics Control Systems for Motorcycle". In: Handbook of Driver Assistance Systems. Ed. by Hermann Winner et al. Vol. 19. Cham: Springer International Publishing, 2014 pp. 1-29. ISBN: 978-3-319-09840-1. DOI: 10.1007/978-3319-09840-1\{ \textunderscore\}42-1.

[27] DIN ISO 8855:2013-11: Straßenfahrzeuge - Fahrzeugdynamik und Fahrverhalten - Begriffe. Berlin. DOI: 10.31030/ 1941260.

[28] GeneSys Elektronik GmbH. "Automotive Testing Equipment: Products 2015". In: (2015). URL: https : / / www . genesys - offenburg . de / produkte / adma familie-gnssinertialsystem-automotivebahn/ (visited on 08/16/2020).

[29] Zhizhong Ma et al. "Experimental Evaluation of Mobile Phone Sensors". In: ISSC 2013. Lytterkenny, 2013-06-20.

[30] Anton Kos, Sašo Tomažič, and Anton Umek. "Evaluation of Smartphone Inertial Sensor Performance for CrossPlatform Mobile Applications". In: Sensors (Basel, Switzerland) 16.4 (2016). DOI: 10.3390/s16040477.

[31] Ivo Boniolo et al. "On the Roll Angle Estimate via Inertial Sensors: Analysis of the Principal Measurement Axes: DSCC2009-2562". In: ASME Dynamic Systems and Control Conference. Hollywood, California: ASME, 2009-10-12.

[32] I. Boniolo and S. M. Savaresi. "Motorcycle lean angle estimation with frequency separation principle and angular rates measurements". In: IFAC Symposium Advances in Automotive Control. Munich, Germany: IFAC, 2010-07-12.

[33] Gabor Ambruzs, Robert Weisz, and David Szaloky. "Method to determine the roll angle of a motorcycle: Patent". WO 2017/149158 Al. 2017-09-08.

[34] Ivo Boniolo et al. "Performance analysis of an optical distance sensor for roll angle estimation in sport motorcycles". In: 17th IFAC World Congress. Seoul, Korea: IFAC, 2008-07-06. DOI: 10.3182/20080706-5-KR-1001.3487. URL: http://arxiv.org/pdf/1001.3487v1.

[35] M. Norgia et al. "Optical Sensors for Real-Time Measurement of Motorcycle Tilt Angle". In: IEEE Transactions on Instrumentation and Measurement 58.5 (2009), pp. 1640 1649. ISSN: 0018-9456. DOI: 10.1109/TIM.2008.2009421.

[36] Roberto Lot, Vittore Cossalter, and Matteo Massaro, eds. Real-time Roll Angle Estimation for two-wheeled vehicles: ESDA2012-82182. s.I.: Lulu, 2012. ISBN: 9781430308614.

[37] Stefano Corbetta, Ivo Boniolo, and Sergio M. Savaresi. "Attitude estimation of a motorcycle via Unscented Kalman Filter". In: 5th IFAC Symposium Mechatronic Systems. Marriott Boston Cambridge, 2010-09-13.
[38] Marc Schlipsing et al. Roll Angle Estimation for Motorcycles: Comparing Video and Inertial Sensor Approaches: 3 - 7 June 2012, Alcalá de Henares, Madrid, Spain. Piscataway, NJ: IEEE, 2012. ISBN: 9781467321181. URL: http://ieeexplore. ieee.org/servlet/opac?punumber=6222409.

[39] S. Staacks et al. "Advanced tools for smartphone-based experiments: phyphox". In: Physics Education 53.4 (2018), p. 045009. ISSN: 0031-9120. DOI: 10.1088/1361-6552/ aac05e.

[40] Inc. Freescale Semiconductor. AN4399 High Precision Calibration of a Three-Axis Accelerometer: Application Note. Rev. 2.0, 10/2015. Oct. 2015.

[41] R. E. Kalman. "A New Approach to Linear Filtering and Prediction Problems". In: Journal of Basic Engineering 82.1 (1960), p. 35. ISSN: 00219223. DOI: 10.1115/1.3662552.

[42] Service de geodesie et nivellement. Projection cartographique Mercator Transverse: SGN27815; Algorithmes. Saint Mande Cedex, 1995-01.

[43] Christian Gorges, Kemal Öztürk, and Robert Liebich "Road classification for two-wheeled vehicles". In: Vehicle System Dynamics 56.8 (2018), pp. 1289-1314. ISSN: 0042-3114. DOI: 10.1080/00423114.2017.1413197.

[44] Statistische Ämter des Bundes und der Länder. Unfallatlas. Wiesbaden, 2019. URL: https : / / unfallatlas. statistikportal.de/ (visited on 07/13/2020).

[45] William T. Neale et al. "Data Acquisition using Smart Phone Applications". In: SAE Technical Paper Series. SAE Technical Paper Series. SAE International, 2016. DOI: 10. 4271/2016-01-1461.

[46] Raphael Pleß et al. "Investigation of the existence of a leaning threshold among motorcyclists". In: 13th International Motorcycle Conference. Köln: IFZ, 2020.

[47] Sebastian Will et al. "Methodological considerations regarding motorcycle naturalistic riding investigations based on the use of g-g diagrams for rider profile detection". In: Safety Science 129 (2020), p. 104840. ISSN: 09257535. DOI: 10.1016/j.ssci.2020.104840.

[48] Tino Staffetius. "Investigations on the behaviour of motorcyclists with different driving experience on test-areas and in real road traffic". In: 11th International Motorcycle Conference. Ed. by IFZ. Köln, 2016.

[49] Matthias Lehmann et al., eds. Use of a criticality metric for assessment of critical traffic situations as part of SePIA. ATZ live. Wiesbaden and [Heidelberg]: Springer Vieweg, 2019. ISBN: 978-3-658-25938-9.

[50] Hiroki Watanabe et al. "Scenario Mining for Development of Predictive Safety Functions". In: IEEE International Conference. 2019, pp. 1-7. DOI: 10.1109/ICVES.2019.8906293.

[51] Volker Quarz, Kay Büttner, and Franka-Maria Volk. “Development of a method for analysis and effectiveness evaluation of driving safety functions in powered twowheeler". In: IFZ International Motorcycle Conference 2014. Köln, 2014 